\title{
Synchronization vs. decoherence of neutrino oscillations at intermediate densities
}

\author{
Georg G. Raffelt ${ }^{1}$ and Irene Tamborra ${ }^{1,2,3}$ \\ ${ }^{1}$ Max-Planck-Institut für Physik (Werner-Heisenberg-Institut), Föhringer Ring 6, 80805 München, Germany \\ ${ }^{2}$ Dipartimento Interateneo di Fisica "Michelangelo Merlin", Via Amendola 173, 70126 Bari, Italy \\ ${ }^{3}$ INFN, Sezione di Bari, Via Orabona 4, 70126 Bari, Italy
}

(Dated: 1 December 2010)

\begin{abstract}
We study collective oscillations of a two-flavor neutrino system with arbitrary but fixed density. In the vacuum limit, modes with different energies quickly de-phase (kinematical decoherence), whereas in the limit of infinite density they lock to each other (synchronization). For intermediate densities, we find different classes of solutions. There is always a phase transition in the sense of partial synchronization occurring only above a density threshold. For small mixing angles, partial or complete decoherence can be induced by a parametric resonance, introducing a new time scale to the problem, the final outcome depending on the spectrum and mixing angle. We derive an analytic relation that allows us to calculate the late-time degree of coherence based on the spectrum alone.

PACS numbers: 14.60.Pq, 97.60.Bw
\end{abstract}

\section{INTRODUCTION}

Flavor oscillations in dense neutrino gases exhibit collective phenomena caused by neutrino-neutrino interactions [1 17]. Recently this topic has been studied intensely in the context of supernova neutrino oscillations; for a recent review see Ref. [18]. In much of this literature, numerical tools were used to study cases of practical interest for supernova neutrinos. Moreover, there has been progress in the analytic understanding of such intriguing phenomena as spectral splits, caused by the adiabatic decrease of the neutrino density with radius in a supernova. However, our theoretical understanding of many aspects of collective oscillations remains unsatisfactory. This applies, in particular, to the question of angular synchronization vs. angular decoherence of neutrinos streaming from a supernova core $11-13]$.

With such general questions in mind, we here return to an elementary case of collective effects: synchronized oscillations in an isotropic neutrino gas with fixed density. Modes with different energies, $E$, and thus different vacuum oscillation frequencies, $\omega=\Delta m^{2} / 2 E$ with $\Delta m^{2}$ the neutrino mass-squared difference, quickly dephase (kinematical decoherence). However, if the density is large one obtains synchronized oscillations: all modes "stick together" and oscillate with a common frequency $\omega_{\text {sync }}=\langle\omega\rangle$ [7]. In the language of flavor-polarization vectors $\mathbf{P}_{\omega}$, all modes together form a common global $\mathbf{P}=\int \mathrm{d} \omega \mathbf{P}_{\omega}$ that precesses as a single object.

While the extreme cases of very dilute and very dense gases are easy to understand, the question of what exactly happens for intermediate densities is less obvious. Different possible answers come to mind. (i) The system could decohere completely, but perhaps on a time scale that grows longer for larger neutrino densities. (ii) A stationary state with some global $\mathbf{P}$ of reduced length could be reached (partial decoherence).

The correct answer has elements of both ideas, in detail depending on the neutrino spectrum, density, and mixing angle. In the simplest case of maximal mixing, the system decoheres completely for densities below a certain threshold value. For larger densities, synchronization is partial and becomes perfect in the limit of infinite density. The behavior of the system as a function of density thus shows a behavior similar to a phase transition.

Other forms of behavior arise for smaller mixing angles. The main reason is that polarization vectors that are initially aligned with each other and almost aligned with the effective magnetic field responsible for their precession can later deviate strongly from both directions due to parametric resonance effects. Depending on whether or not the resonance frequency falls within the spectrum, complete decoherence can arise, but can take a very long time to reach equilibrium. In other words, the decoherence time scale can be very different from any other time scale of the problem.

Our study is in many ways complementary to Pantaleone's investigation of the Stability of incoherence in an isotropic gas of oscillating neutrinos [2]. He asked if a completely incoherent initial state would spontaneously develop global flavor polarization, a process resembling the spontaneous synchronization of a collection of coupled oscillators. He found neutral stability, implying that spontaneous synchronization does not occur, largely because the equations of motions conserve energy. This contrasts with some models designed to mimic spontaneous synchronization of certain biological systems [2].

Returning to the polarization decay of an initially synchronized neutrino ensemble, we begin in Sec. II by setting up the equations of motion of a system of interacting flavor polarization vectors. In Sec. III we consider simple cases of kinematical decoherence. In Sec.[IV we derive analytic relations that allow us to calculate the asymptotic behavior. In Sec. $\mathrm{V}$ we show that the threshold behavior of partial synchronization can be understood if one represents the spectrum in terms of two discrete polarization vectors. In Sec. VI we identify the phenomenon of selfinduced resonant decoherence. In Sec. VII we summarize our findings. 


\section{EQUATIONS OF MOTION}

\section{A. Matrices of occupation numbers}

For the kinetic evolution of a particle ensemble, the fundamental entities are occupation numbers $f_{\mathbf{p}}=$ $\left\langle a_{\mathbf{p}}^{\dagger} a_{\mathbf{p}}\right\rangle$ for every mode $\mathbf{p}$. Here $a_{\mathbf{p}}^{\dagger} a_{\mathbf{p}}$ is a number operator and $\langle\cdots\rangle$ an expectation value or ensemble average. Including the flavor degree of freedom, the occupation numbers are replaced by flavor matrices of occupation numbers $\varrho_{\mathbf{p}, \alpha \beta}=\left\langle a_{\mathbf{p}, \alpha}^{\dagger} a_{\mathbf{p}, \beta}\right\rangle$ where $\alpha$ and $\beta$ are flavor indices. The diagonal entries represent the occupation numbers for different flavors and are related to the survival probabilities, whereas the off-diagonal elements encode phase information [19, 20]. Based on this formalism, one can derive a Boltzmann collision equation that allows one to study the evolution of a neutrino ensemble under the influence of both flavor oscillations and collisions [19, 20].

Here we study a much simpler case where collisions are neglected, motivated by neutrinos streaming freely from a supernova core. However, in our schematic model we ignore all geometric effects and consider the time evolution of an isotropic ensemble rather than the spatial variation of a stationary flux. In the limit of ultrarelativistic neutrinos, the equations of motion (EoMs) are [20]

$$
\mathrm{i} \dot{\varrho}_{\mathbf{p}}=\left[\frac{M^{2}}{2 E}+\sqrt{2} G_{\mathrm{F}} \varrho, \varrho_{\mathbf{p}}\right],
$$

where $[\cdot, \cdot]$ is a commutator and $M^{2}$ is the neutrino mass-squared matrix. It is not diagonal in the weak interaction basis, causing vacuum flavor oscillations. The global matrix $\varrho=\int \varrho_{\mathbf{p}} \mathrm{d}^{3} \mathbf{p} /(2 \pi)^{3}$ has diagonal entries of neutrino number densities. This "neutrino matter term" has the same structure as the ordinary matter term where the role of the global $\varrho$ is played by the matrix of electron, muon and tau-lepton number densities that is diagonal in the weak interaction basis. Indeed, a background of neutrinos would be fully analogous to ordinary matter, were it not for the off-diagonal elements of $\varrho$ as first recognized in Pantaleone's seminal paper [1].

Full kinematical decoherence corresponds to the offdiagonal elements of $\varrho$ vanishing in the mass basis. At a sufficient distance from a source, a neutrino flux will always end up in such a state. It is sometimes described as the wave packets of different mass eigenstates no longer overlapping.

\section{B. Polarization vectors}

For many studies it proves more transparent to represent $\varrho_{\mathbf{p}}$ in terms of polarization vectors $\mathbf{P}_{\mathbf{p}}$ in "flavor space." We restrict our investigation to two flavors where $\varrho_{\mathbf{p}}$ is a Hermitean $2 \times 2$ matrix and thus can be expressed in terms of Pauli matrices as $\varrho_{\mathbf{p}}=\frac{1}{2} \operatorname{Tr}\left(\varrho_{\mathbf{p}}\right)+\frac{1}{2} \mathbf{P}_{\mathbf{p}} \cdot \boldsymbol{\sigma}$ (while for three flavors, we need Gell-Mann matrices and 8-dimensional polarization vectors). Likewise, we may write $M^{2} / 2 E=\frac{1}{2} \operatorname{Tr}\left(M^{2} / 2 E\right)+\frac{1}{2} \omega \mathbf{B} \cdot \boldsymbol{\sigma}$ where $\omega=\Delta m^{2} / 2 E$ is the vacuum oscillation frequency and B a unit vector, playing the role of an effective magnetic field in the spin-precession formula below.

We study an isotropic ensemble, i.e. we consider modes $\varrho_{E}$ that represent all momentum modes of the same energy $E=|\mathbf{p}|$. It proves more convenient to label the modes with $\omega=\Delta m^{2} / 2 E$ and corresponding polarization vectors $\mathbf{P}_{\omega}$. The EoMs then appear in the familiar precession form (Fig. 1)

$$
\dot{\mathbf{P}}_{\omega}=\mathbf{H}_{\omega} \times \mathbf{P}_{\omega} \quad \text { where } \quad \mathbf{H}_{\omega}=\omega \mathbf{B}+\mu \mathbf{P} .
$$

The total polarization vector is $\mathbf{P}=\int \mathrm{d} \omega \mathbf{P}_{\omega}$ which we normalize as $|\mathbf{P}|=1$. In this case $\mu=\sqrt{2} G_{\mathrm{F}} n_{\nu}$, with $n_{\nu}$ the neutrino number density, is the interaction energy felt by a neutrino in the mean field of all others.

The precession of individual modes is around $\mathbf{B}$ which thus marks a natural symmetry direction of the problem. Therefore, we choose the $\mathbf{B}$-direction as the $z$-direction of a cartesian coordinate system in flavor space. We assume that all neutrinos are initially prepared in the same flavor, so all $\mathbf{P}_{\omega}$ initially point in the weak-interaction direction. It is tilted relative to $\mathbf{B}$ by twice the mixing angle $\theta$ (Fig. 11). Therefore, we have

$$
\mathbf{B}=\left(\begin{array}{l}
0 \\
0 \\
1
\end{array}\right) \quad \text { and } \quad \mathbf{P}_{\omega}(0)=\left(\begin{array}{c}
\sin 2 \theta \\
0 \\
\cos 2 \theta
\end{array}\right) g_{\omega}
$$

where $g_{\omega}=g(\omega)=\left|\mathbf{P}_{\omega}(0)\right|$ is what we call the neutrino spectrum normalized to $\int d \omega g_{\omega}=1$. The initial direction in the $x-y$-plane is arbitrary, owing to a global $\mathrm{SU}(2)$ symmetry of the problem: the absolute phase of $\nu_{e}$ relative to another flavor $\nu_{x}$ is arbitrary.

The EoMs conserve the quantity $\int \mathrm{d} \omega \omega \mathbf{B} \cdot \mathbf{P}_{\omega}+\frac{1}{2} \mu \mathbf{P}^{2}$ which thus plays the role of total energy. This is seen by

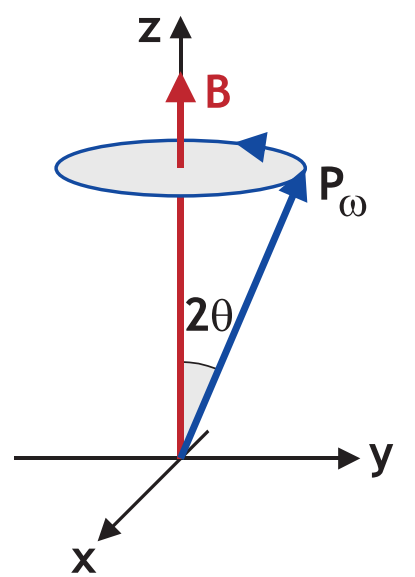

FIG. 1: Precession of a polarization vector $\mathbf{P}_{\omega}$ with frequency $\omega$ around the $z$-direction (the mass direction in flavor space). The initial orientation is in the weak-interaction direction, tilted by twice the mixing angle relative to the $z$-direction. 
taking the time derivative and inserting the EoMs on the r.h.s. for $\dot{\mathbf{P}}_{\omega}$. In other words, the EoMs can be derived from a classical Hamiltonian. This is seen most easily if we use discrete polarization vectors $\mathbf{P}_{i}$ for a set of $N$ frequency bins $\omega_{i}$ where $\mathbf{P}=\sum_{i} \mathbf{P}_{i}$,

$$
H=\sum_{i=1}^{N} \omega_{i} \mathbf{B} \cdot \mathbf{P}_{i}+\frac{\mu}{2} \sum_{i, j=1}^{N} \mathbf{P}_{i} \cdot \mathbf{P}_{j} .
$$

The polarization vectors play the role of classical angular momenta with Poisson brackets $\left[P_{x}, P_{y}\right]=P_{z}$ and cyclic permutations. ${ }^{1}$ They inherit this property from the $\mathrm{SU}(2)$ algebra of the "flavor isospin matrices" [10]. The precession equations follow from the classical Hamilton equations of motion $\dot{\mathbf{P}}_{i}=\left[\mathbf{P}_{i}, H\right]$. The $\mathbf{P}_{i}$ are classical variables because they arise from occupation number matrices which themselves are classical objects, not quantum operators, as they represent an expectation value.

The quantity $\mathbf{P}$ plays the role of the total angular momentum of the ensemble. Naturally, its component along $\mathbf{B}$ is conserved. The conservation of $\mathbf{B} \cdot \mathbf{P}$ is also easily seen by taking the time derivative of this bilinear and inserting the EoMs for $\dot{\mathbf{P}}_{\omega}$.

In the polarization-vector language, our ensemble of interacting neutrinos is equivalent to an ensemble of classical angular momenta $\mathbf{P}_{i}$ that couple to an external magnetic field with strength $\omega_{i}$ and to each other with a dipole-dipole interaction of equal strength $\mu$. However, they do not couple by some nearest-neighbor interaction, but rather every mode couples to every other with the same strength.

\section{Antineutrinos}

Occupation number matrices for antineutrinos can be defined in three different ways, all of which have been used in the literature. If $b_{\mathbf{p}}^{\dagger}$ and $b_{\mathbf{p}}$ are creation and annihilation operators for antineutrinos in mode $\mathbf{p}$ we may define $\bar{\varrho}_{\mathbf{p}, \alpha \beta}=\left\langle b_{\mathbf{p}, \alpha}^{\dagger} b_{\mathbf{p}, \beta}\right\rangle$ or $\left\langle b_{\mathbf{p}, \beta}^{\dagger} b_{\mathbf{p}, \alpha}\right\rangle$ or $1-\left\langle b_{\mathbf{p}, \beta}^{\dagger} b_{\mathbf{p}, \alpha}\right\rangle$, the latter arising from normal ordering of $\left\langle b_{\mathbf{p}, \alpha} b_{\mathbf{p}, \beta}^{\dagger}\right\rangle$. The third definition amounts to the flavor-isospin interpretation of polarization vectors [10] and is the most useful in our context. Antiparticles are interpreted as negative-energy states and are thus described by $\omega=\Delta m^{2} / 2 E<0$. In the polarization-vector language, antineutrinos are then described by $\mathbf{P}_{\omega}$ with negative $\omega$.

The flavor interpretation is now as follows: $\mathbf{P}_{\omega}$ in the positive weak-interaction direction means $\nu_{e}$, in the negative weak-interaction direction means $\nu_{x}$ if $\omega>0$, whereas for $\omega<0$ these cases mean $\bar{\nu}_{x}$ and $\bar{\nu}_{e}$, respectively. This sounds complicated, but is simple in the hole

\footnotetext{
${ }^{1}$ Here $[\cdot, \cdot]$ is a Poisson bracket, not a commutator. Of course, classical variables commute in the sense of $P_{x} P_{y}=P_{y} P_{x}$.
}

interpretation of antiparticles where the presence of $\bar{\nu}_{e}$ in mode $\mathbf{p}$ is equivalent to the absence of a negative-energy $\nu_{e}$ from a filled mode in the Dirac sea.

Once we are studying the dynamics of our abstract Hamiltonian problem of interacting spins, the detailed flavor interpretation is irrelevant. We simply consider an ensemble of $\mathbf{P}_{\omega}$ with a range of positive and negative $\omega$.

\section{Rotating frames}

The dynamics of the system is the same if we go to a frame rotating with $\omega_{0}$ around the $z$-direction [2, 9]. This is seen most easily if we consider the spin-spin Hamiltonian of Eq. (4) and recall that $\mathbf{B} \cdot \mathbf{P}$ is conserved. Therefore, we may add a term $-\omega_{0} \mathbf{B} \cdot \mathbf{P}$, leading to

$$
H=\sum_{i=1}^{N}\left(\omega_{i}-\omega_{0}\right) \mathbf{B} \cdot \mathbf{P}_{i}+\frac{\mu}{2} \sum_{i, j=1}^{N} \mathbf{P}_{i} \cdot \mathbf{P}_{j} .
$$

Therefore, even if we are having in mind an ensemble of neutrinos (no antineutrinos) and therefore a range of positive $\omega$, we may shift the spectrum $g(\omega)$ by a convenient amount $-\omega_{0}$ and thus include negative $\omega$. In other words, the negative- $\omega$ part of the spectrum can be interpreted as antineutrino modes or as neutrino modes after such a shift. The possibility of this general interpretation and the seamless connection of neutrino and antineutrino modes is the main advantage of the flavor-isospin interpretation of polarization vectors.

\section{E. Spectra}

We always consider non-negative spectra, $g_{\omega} \geq 0$, avoiding "spectral crossings." The latter lead to unstable forms of motion ("flavor pendulum" 14]) that are at the origin of spectral splits [17]. Addressing the question of decoherence in such more general cases is beyond our present ambition.

We will explicitly study two cases of simple spectra ${ }^{2}$ that are symmetric about $\omega=0$ and normalized to unity. One is a box spectrum where $g_{\omega}=0$ everywhere except

$$
g_{\omega}=\frac{1}{2} \quad \text { for } \quad-1<\omega<+1 .
$$

The other example is a Gaussian with unit variance

$$
g_{\omega}=\frac{1}{\sqrt{2 \pi}} \exp \left(-\frac{\omega^{2}}{2}\right) .
$$

We show these spectra in Fig. 2 ,

\footnotetext{
2 To simplify notation we take frequencies and time to be dimensionless, or rather, to be expressed in terms of some fiducial frequency $\omega_{0}$ so that $\omega \rightarrow \omega / \omega_{0}, \mu \rightarrow \mu / \omega_{0}$, and $t \rightarrow \omega_{0} t$.
} 


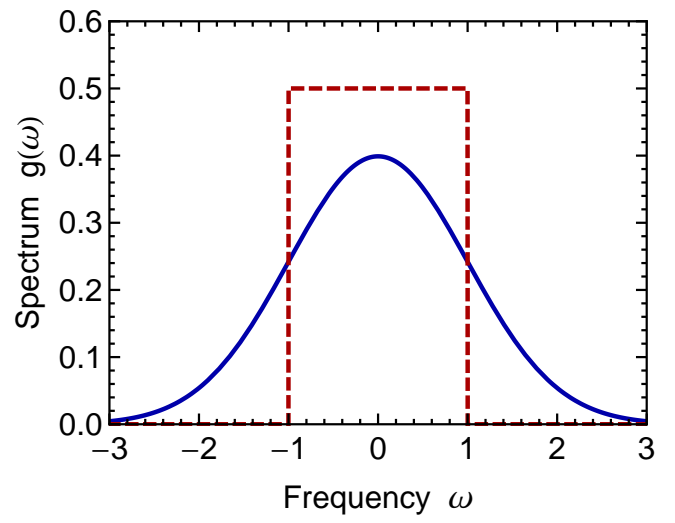

FIG. 2: Box spectrum (red, dashed) and Gaussian spectrum (blue, solid) as defined in Eqs. (6) and (7) respectively.

The box spectrum is representative for "compact" spectra that are non-zero in a finite $\omega$-range, whereas the Gaussian is representative for spectra with long tails. We will see that spectral tails are crucial for the phenomenon of resonant decoherence to be discussed in Sec. VI

\section{KINEMATICAL DECOHERENCE}

\section{A. Order Parameter}

When neutrino modes de-phase relative to each other, the system becomes in some sense disordered, but it is not trivial to define an objective measure for this effect. Flavor oscillations alone (no collisions) do not increase the entropy of the system [20]. Kinematical decoherence is reversible. For example, a neutrino flux decoheres kinematically after a short distance from the source, but a detector with sufficient energy resolution can still pick out oscillations in an energy-dependent way and in this sense restores coherence. Another example is the phenomenon of spin echo: reversing the direction of the magnetic field unwinds the phases accrued by different modes and the original polarization re-appears.

In our case the simplest quantity to measure coherence is what Pantaleone calls the "order parameter" [2]

$$
R(t)=\frac{\left|\mathbf{P}_{\perp}(t)\right|}{\sin 2 \theta}
$$

where it was assumed that $|\mathbf{P}(0)|=1$. Here $\mathbf{P}_{\perp}$ is that part of $\mathbf{P}$ transverse to $\mathbf{B}$. Since $\mathbf{B} \cdot \mathbf{P}$ is conserved, it is only the transverse component that can shrink by kinematical decoherence. Dividing by $\sin 2 \theta$ normalizes the initial order parameter to $R(0)=1$.

Were we to study decoherence for more complicated spectra, this definition would be less useful. When spectral crossings and pendular motions are involved, $\mathbf{P}_{\perp}$ can grow even though the system becomes "more disordered" as it evolves. Probably one would need to define kinematical decoherence in a differential sense, the dephasing of

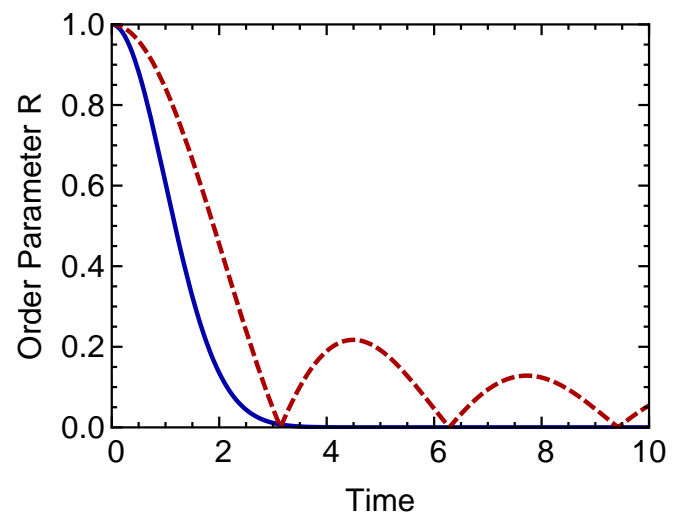

FIG. 3: Decay of the order parameter $R(t)$, defined in Eq. (8), for the box spectrum (red, dashed) and the Gaussian spectrum (blue, solid) as given in Eq. (10).

neighboring modes, leading to a shrinking of $\mathbf{P}_{\omega}$ after coarse-graining over small but finite frequency bins $\Delta \omega$. For our simple cases we are using a single frequency bin: the entire spectrum.

\section{B. Decoherence in the non-interacting case}

De-phasing is easy to understand in the dilute limit where neutrino-neutrino interactions play no role $(\mu=0)$ and the evolution is determined by vacuum oscillations alone. The transverse components evolve as $P_{x}(t)=$ $\sin 2 \theta \int \mathrm{d} \omega g_{\omega} \cos (\omega t)$ and $P_{y}(t)=\sin 2 \theta \int \mathrm{d} \omega g_{\omega} \sin (\omega t)$. In other words, the order parameter evolves as

$$
R(t)=\left|\int \mathrm{d} \omega g_{\omega} \mathrm{e}^{\mathrm{i} \omega t}\right|
$$

Therefore, $R(t)$ is given by the Fourier transform of $g_{\omega}$. Unless $g_{\omega}$ includes "spikes" of the form $\delta\left(\omega-\omega_{i}\right)$ at one or more frequencies $\omega_{i}$, the late-time evolution is $R(t) \rightarrow 0$ for $t \rightarrow \infty$ (complete kinematical decoherence).

For our two explicit examples, the time evolution of the order parameter is (Fig. 3)

$$
\begin{array}{ll}
\text { Box: } & R(t)=\sin (t) / t, \\
\text { Gauss: } & R(t)=\exp \left(-t^{2} / 2\right) .
\end{array}
$$

The box spectrum has "hard edges" and thus shows the usual "ringing" of its Fourier transform.

\section{Self-maintained coherence}

For non-zero interaction strength, the system can show "self-maintained coherence" in that an oscillatory motion persists at late times. We illustrate this in Fig. 4 where we show the evolution of the order parameter $R(t)$ for the Gaussian spectrum with maximal mixing and different values of $\mu$. For $\mu$ above a threshold value $\mu_{0}$ 


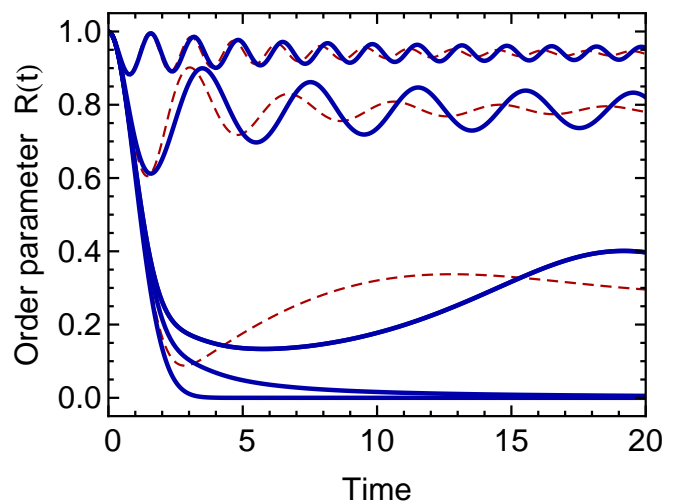

FIG. 4: Order parameter for the Gaussian spectrum with maximal mixing and $\mu=0, \mu_{0}, 1,2$ and 4 (bottom to top), where $\mu_{0} \approx 0.7979$ is the threshold value for partial coherence. Dashed lines: Solution when $\mathbf{P}(t)$ in the EoMs is replaced with the asymptotic solution $\mathbf{A}(t)$ such as if $\mathbf{P}(t)$ would take on its asymptotic solution instantaneously.

to be identified later, $R(t)$ at first shrinks like in the non-interacting case, but then oscillates with decreasing amplitude around a non-vanishing asymptotic value $R_{A}$. In other words, the global polarization vector $\mathbf{P}(t)$ shrinks in length and eventually reaches a nonvanishing asymptotic form $\mathbf{A}(t)$ that keeps precessing around the $\mathbf{B}$ direction with a certain asymptotic frequency $\omega_{A}$. Of course, in the extreme case $\mu \rightarrow \infty$ we have perfectly synchronized oscillations and the asymptotic length is $|\mathbf{A}(t)|=|\mathbf{P}(0)|=1$ and $\omega_{A}=\omega_{\text {sync }}=\langle\omega\rangle$.

An asymptotic solution $\mathbf{P}(t) \rightarrow \mathbf{A}(t)$ performing a pure precession around $\mathbf{B}$ is the simplest case of collective motion. We may call it single-mode coherence in that the asymptotic solution is described by a single asymptotic polarization vector.

However, we may construct more complicated spectra $g(\omega)$ where the asymptotic solution contains two or more modes. For example, if $g(\omega)$ consists of two separate box spectra, then for vanishing $\mu$ the spectrum completely decoheres and for infinite $\mu$ the system acts as one synchronized mode. However, one can choose $\mu$ so large that each individual box acts as one collective mode, being internally synchronized, yet so small that each box essentially precesses freely around $\mathbf{B}$. We now have effectively two weakly coupled modes and thus bimodal coherence. In the same way we can construct a hair-comb spectrum consisting of $N$ boxes, leading for intermediate $\mu$ to $N$ modal coherence.

A priori it is not obvious if a given spectrum, for a given interaction strength $\mu$, will decohere or not, and in the latter case, how many modes are required to describe the asymptotic behavior. In the rest of the paper we study only cases of single-mode coherence where the final behavior is pure precession. This is the case for both the box and the Gaussian spectrum.

\section{Sudden approximation}

We can qualitatively understand the behavior in Fig. 4 if we imagine that $\mathbf{P}(t)$ shrinks instantaneously to its asymptotic form $\mathbf{A}(t)$, an assumption that we call "sudden approximation." We can use the numerical asymptotic solution and evolve the initial state with $\mathbf{A}(t)$ instead of $\mathbf{P}(t)$ in the EoMs, in this way reproducing what we would get if the sudden approximation were indeed correct. We show examples as thin dashed lines in Fig. 4.

In the sudden approximation, kinematical decoherence is once more a passive de-phasing effect except that the individual modes $\mathbf{P}_{\omega}$ no longer precess around a common $\mathbf{B}$, but each precesses around its individual $\mathbf{H}_{\omega}(t)=\omega \mathbf{B}+\mu \mathbf{A}(t)$. There is no feedback of $\mathbf{P}_{\omega}$ on $\mathbf{H}_{\omega}$ and we can determine $R(t)$ by projecting the evolution of the individual modes on the $x$ - $y$-plane. For a symmetric spectrum with maximal mixing we find

$$
R(t)=\int \mathrm{d} \omega g_{\omega} \frac{\kappa^{2}+\omega^{2} \cos \left(\sqrt{\kappa^{2}+\omega^{2}} t\right)}{\kappa^{2}+\omega^{2}},
$$

where $\kappa=\mu A_{\perp}$ which for maximal mixing is $\kappa=\mu A$. For $\kappa=\mu A=0$ this reduces to Eq. (9). For small $t$ we may expand the cosine in Eq. (11) and find $R(t)=$ $1-\frac{1}{2}\left\langle\omega^{2}\right\rangle t^{2}$. The initial evolution depends only on the variance of the spectrum, but not on $\mu$.

\section{PARTIAL SYNCHRONIZATION}

\section{A. Self-consistency conditions}

We next study quantitatively the conditions for the existence of a nonvanishing asymptotic solution $\mathbf{P}(t) \rightarrow$ $\mathbf{A}(t)$ which we assume to be a pure precession with frequency $\omega_{A}$. Conditions for the existence of a nontrivial solution for $\mu<\infty$ can be formulated if we study the system from the perspective of a frame that co-rotates with $\mathbf{A}$ in analogy to the approach taken to study spectral splits [15, 16]. The assumed final steady-state EoM is given by Eq. (2) with

$$
\mathbf{H}_{\omega}=\left(\omega-\omega_{A}\right) \mathbf{B}+\mu \mathbf{A},
$$

where in the co-rotating frame $\mathbf{A}$ is a static vector. The evolution of every individual mode is now a simple precession around this fixed vector and we refer to these asymptotic modes as $\mathbf{A}_{\omega}(t)$.

At late times, these modes must have de-phased, meaning that their oscillation phase relative to their $\mathbf{H}_{\omega}$ carries no correlation with each other. Therefore, the total polarization vector is given by the time averages of $\mathbf{A}_{\omega}$ as $\mathbf{A}=\int \mathrm{d} \omega\left\langle\mathbf{A}_{\omega}\right\rangle$. The time-average of $\mathbf{A}_{\omega}$ is the projection on its Hamiltonian vector

$$
\left\langle\mathbf{A}_{\omega}\right\rangle=\frac{\mathbf{A}_{\omega} \cdot \mathbf{H}_{\omega}}{\mathbf{H}_{\omega}^{2}} \mathbf{H}_{\omega}
$$


implying

$$
\mathbf{A}=\int \mathrm{d} \omega \frac{\mathbf{A}_{\omega} \cdot\left[\left(\omega-\omega_{A}\right) \mathbf{B}+\mu \mathbf{A}\right]}{\left[\left(\omega-\omega_{A}\right) \mathbf{B}+\mu \mathbf{A}\right]^{2}}\left[\left(\omega-\omega_{A}\right) \mathbf{B}+\mu \mathbf{A}\right] .
$$

Recall that the $\mathbf{A}_{\omega}$ in this equation are time dependent, precessing around the asymptotic $\mathbf{H}_{\omega}$.

The quantity $P_{\|}=\cos 2 \theta$ is conserved and therefore identical with $A_{\|}$. It will be useful to introduce

$$
\kappa=\mu A_{\perp} \quad \text { and } \quad \omega_{\mathrm{r}}=\omega_{A}-\mu \cos 2 \theta,
$$

where $\omega_{\mathrm{r}}$ plays the role of a resonance frequency. The components of the self-consistency relation perpendicular and parallel to $\mathbf{B}$ become

$$
\begin{aligned}
\frac{1}{\mu} & =\int \mathrm{d} \omega \frac{\left(\omega-\omega_{\mathrm{r}}\right) A_{\omega \|}+\kappa A_{\omega \perp}}{\left(\omega-\omega_{\mathrm{r}}\right)^{2}+\kappa^{2}}, \\
A_{\|} & =\int \mathrm{d} \omega \frac{\left(\omega-\omega_{\mathrm{r}}\right) A_{\omega \|}+\kappa A_{\omega \perp}}{\left(\omega-\omega_{\mathrm{r}}\right)^{2}+\kappa^{2}}\left(\omega-\omega_{\mathrm{r}}\right),
\end{aligned}
$$

where $\kappa$ and $\omega_{\mathrm{r}}$ are the quantities to be determined.

What is the meaning of the resonance? A given $\mathbf{P}_{\omega}$ precesses in the co-rotating frame with the frequency $\omega-\omega_{A}+\mu \cos 2 \theta$. When this quantity vanishes, $\mathbf{P}_{\omega}$ does not precess around the $z$-direction and only feels the transverse field. This is similar to spin resonance in a longitudinal $B$ field and a rotating transverse field. If the transverse field is on resonance, the spin can completely flip even if the transverse field is weak. We will see that this resonance effect is indeed important for small mixing angles if $\omega_{\mathrm{r}}$ falls within the range of the spectrum.

\section{B. Sudden approximation}

The final angle between $\mathbf{A}_{\omega}$ and the corresponding asymptotic $\mathbf{H}_{\omega}$ is not known, so we need to make additional assumptions. Taking the non-interacting case as a guide, decoherence happens on a timescale of the same order as the individual oscillation frequencies and thus is not adiabatic. Therefore, we use the sudden approximation where the initial $\mathbf{P}$ shrinks instantaneously to the final A. We will see that this heuristic procedure leads to an apparently very general result even though the sudden approximation can be badly violated.

We thus evaluate the integrand in Eq. (16) at time $t=0$, assuming that at this time $\mathbf{A}_{\omega}$ coincides with the initial $\mathbf{P}_{\omega}$ so that $A_{\omega \perp}=g_{\omega} \sin 2 \theta$ and $A_{\omega \|}=g_{\omega} \cos 2 \theta$. With the notation $s=\sin 2 \theta$ and $c=\cos 2 \theta$ we find

$$
\begin{aligned}
\frac{1}{\mu} & =\int \mathrm{d} \omega g_{\omega} \frac{\left(\omega-\omega_{\mathrm{r}}\right) c+\kappa s}{\left(\omega-\omega_{\mathrm{r}}\right)^{2}+\kappa^{2}} \\
c & =\int \mathrm{d} \omega g_{\omega} \frac{\left(\omega-\omega_{\mathrm{r}}\right) c+\kappa s}{\left(\omega-\omega_{\mathrm{r}}\right)^{2}+\kappa^{2}}\left(\omega-\omega_{\mathrm{r}}\right) .
\end{aligned}
$$

One can easily show that in the limit $\mu \rightarrow \infty$ the equations are solved by $A_{\perp}=s$ and $\omega_{A}=\omega_{\text {sync }}=\int \mathrm{d} \omega g_{\omega} \omega$, the usual synchronized oscillations.
One can form linear combinations of these equations and also use $1=\int \mathrm{d} \omega g_{\omega}$. One interesting form is

$$
1=\int \mathrm{d} \omega g_{\omega} \frac{\left(1+t^{2}\right) R_{A}}{\xi^{2}+t^{2} R_{A}^{2}}=\int \mathrm{d} \omega g_{\omega} \frac{\left(1+t^{2}\right) \xi}{\xi^{2}+t^{2} R_{A}^{2}},
$$

where $\xi=\left(\omega-\omega_{\mathrm{r}}\right) / \mu c=\left(\omega-\omega_{A}\right) / \mu c+1$ and $t=\tan 2 \theta=$ $s / c$. We recall that $R_{A}=A_{\perp} / \sin 2 \theta$ is the asymptotic value of the order parameter with $0 \leq R_{A} \leq 1$.

\section{Analytic properties}

Perhaps the most illuminating form of the relations is

$$
\begin{aligned}
\frac{s}{\mu} & =\int \mathrm{d} \omega g_{\omega} \frac{\kappa}{\left(\omega-\omega_{\mathrm{r}}\right)^{2}+\kappa^{2}}, \\
\frac{c}{\mu} & =\int \mathrm{d} \omega g_{\omega} \frac{\left(\omega-\omega_{\mathrm{r}}\right)}{\left(\omega-\omega_{\mathrm{r}}\right)^{2}+\kappa^{2}} .
\end{aligned}
$$

We may combine them to a single equation by adding the first, multiplied with the imaginary unit $i$, to the second. Using $\left(\omega-\omega_{\mathrm{r}}+\mathrm{i} \kappa\right)\left(\omega-\omega_{\mathrm{r}}-\mathrm{i} \kappa\right)$ for the denominator it yields

$$
\frac{\mathrm{e}^{\mathrm{i} 2 \theta}}{\mu}=\int \mathrm{d} \omega \frac{g_{\omega}}{\omega-\omega_{\mathrm{r}}-\mathrm{i} \kappa},
$$

where $\omega_{\mathrm{r}}$ is real and $\kappa$ is real and positive.

Going one step further, we add an imaginary part to $g(\omega)$ and extend it to the complex plane by ${ }^{3}$

$$
\mathrm{G}(\Omega)=\frac{1}{\mathrm{i} \pi} \int_{-\infty}^{+\infty} \mathrm{d} \omega^{\prime} \frac{g\left(\omega^{\prime}\right)}{\omega^{\prime}-\Omega}
$$

This complex-valued spectrum in the complex plane fulfills the boundary condition $g(\omega)=\operatorname{Re} G(\omega)$ and obeys Kramers-Kronig relations. The self-consistency relations are now expressed in the compact form

$$
\mu^{-1} \mathrm{e}^{\mathrm{i} 2 \theta}=\mathrm{i} \pi \mathrm{G}\left(\omega_{\mathrm{r}}+\mathrm{i} \kappa\right) .
$$

This analytic relation suggests that our heuristic argument has provided a more general result than justified by its derivation. The predicted asymptotic solution actually agrees with all numerical examples even when the sudden approximation is strongly violated.

For our main examples, the extended spectra are found by direct integration

$$
\begin{aligned}
& \text { Box: } \quad \mathrm{G}(\Omega)=\frac{\mathrm{i}}{2 \pi} \log \left(\frac{\Omega+1}{\Omega-1}\right), \\
& \text { Gauss: } \mathrm{G}(\Omega)=\frac{1}{\sqrt{2 \pi}} \exp \left(-\frac{\Omega^{2}}{2}\right) \operatorname{erfc}\left(-\mathrm{i} \frac{\Omega}{\sqrt{2}}\right),
\end{aligned}
$$

where $\operatorname{erfc}(x)=1-\operatorname{erf}(x)$ and erf is the error function. The box case can be inverted, providing explicitly

$$
\omega_{\mathrm{r}}+\mathrm{i} \kappa=-\operatorname{coth}\left(\frac{\mathrm{e}^{\mathrm{i} 2 \theta}}{\mu}\right) .
$$

\footnotetext{
${ }^{3}$ We use capital sans-serif letters to denote complex quantities.
} 


\section{Threshold for partial synchronization}

Even without explicit evaluation of $G(\Omega)$, we recognize that a minimal interaction strength $\mu_{0}$ is required to achieve partial synchronization. We consider vanishing asymptotic $A_{\perp}$ and thus the $\kappa=0$ limit. The second integral in Eq. (19) becomes

$$
\frac{c}{\mu}=\int \mathrm{d} \omega \frac{g_{\omega}}{\omega-\omega_{0}},
$$

where $\omega_{0}$ is the resonance frequency corresponding to $\kappa=0$ and the integral is to be evaluated in the principalvalue sense. Thus one can determine $\omega_{0}$ and use it to evaluate the first integral where for $\kappa \rightarrow 0$ the second factor becomes $\pi \delta\left(\omega-\omega_{0}\right)$. Therefore we find that

$$
\mu_{0}=\frac{\sin 2 \theta}{\pi g\left(\omega_{0}\right)}
$$

is required to obtain partial synchronization. For $\mu<\mu_{0}$ one expects complete decoherence, although the equations may have formal solutions with negative $A_{\perp}$.

For the box spectrum one finds an explicit result for any mixing angle, whereas for the Gaussian spectrum only for maximal mixing,

$$
\begin{array}{ll}
\text { Box: } & \mu_{0}=(2 / \pi) s, \\
\text { Gauss: } & \mu_{0}=\sqrt{2 / \pi} \text { for } s=1 .
\end{array}
$$
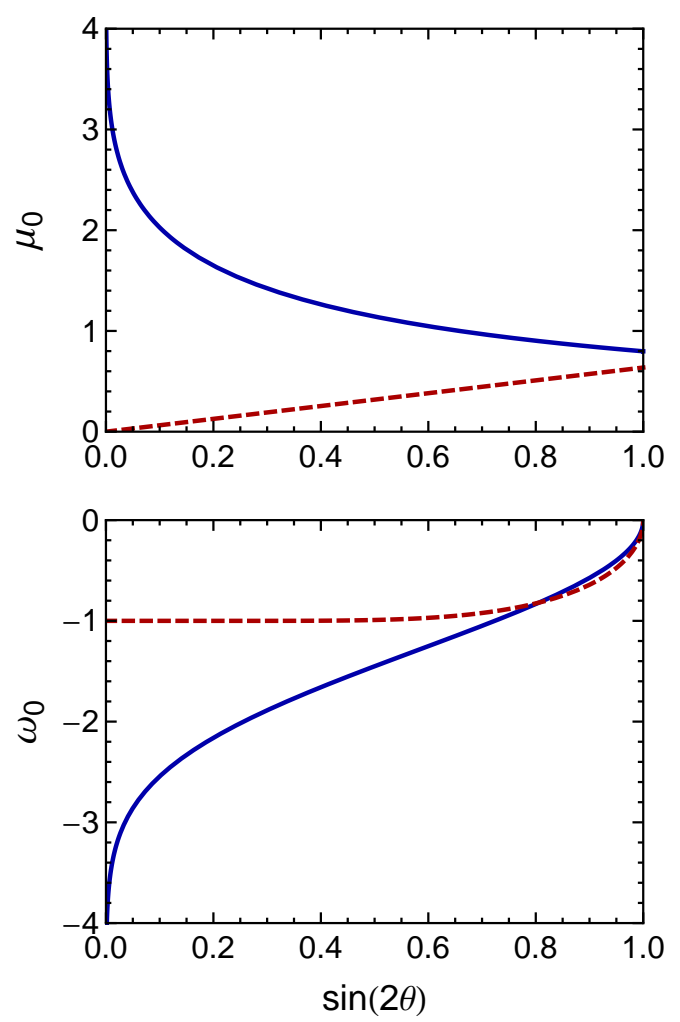

FIG. 5: Threshold interaction strength $\mu_{0}$ and threshold resonance frequency $\omega_{0}$ for the Gaussian (solid blue) and the box spectrum (red dashed).
For general $s$, we use Eq. (22) with $\kappa=0$ and threshold resonance frequency $\omega_{0}$ to obtain the parametric solution $s_{0}\left(\omega_{0}\right)$ and $\mu_{0}\left(\omega_{0}\right)$ with

$$
\begin{aligned}
s_{0} & =\sin \left\{\arg \left[\mathrm{i} G\left(\omega_{0}\right)\right]\right\} \\
\mu_{0} & =\left|\pi \mathrm{G}\left(\omega_{0}\right)\right|^{-1} .
\end{aligned}
$$

Using the explicit $G(\omega)$ for the Gaussian it is straightforward to plot $\mu_{0}$ as a function of $\sin 2 \theta$ in Fig. 5. For small $\sin 2 \theta$, the Gaussian case behaves completely differently due to resonant decoherence as we will explain in Sec.VI.

These results for $0 \leq \sin 2 \theta \leq 1(0 \leq \theta \leq \pi / 4)$ cover only the inverted hierarchy. In this sense it would have been better to consider the range $0 \leq \theta \leq \pi / 2$ and use $-1 \leq \cos 2 \theta \leq+1$ as a mixing variable. For a symmetric spectrum, however, the results are the same except that $\omega_{r} \rightarrow-\omega_{r}$ in the normal hierarchy.

\section{ORIGIN OF THRESHOLD}

\section{A. Maximal Mixing and symmetric spectrum}

The component of $\mathbf{P}$ parallel to $\mathbf{B}$ is conserved and only the transverse component decreases by decoherence. Therefore, to understand the origin of the interaction threshold for partial synchronization we study maximal mixing where $P_{\|}=\cos 2 \theta=0$ and $\sin 2 \theta=1$. To simplify matters further, we assume a symmetric spectrum $g(-\omega)=g(+\omega)$. As a consequence, $\omega_{\mathrm{A}}=\omega_{\mathrm{r}}=0$ for any $\mu$, i.e. the global polarization vector remains static except for its length $P(t)$ and the problem reduces to finding this function.

With $\omega_{A}=0$ and $\kappa=\mu A_{\perp}($ which here is $\kappa=\mu A)$, we need only one real-valued self-consistency relation

$$
\frac{1}{\mu}=\int \mathrm{d} \omega g_{\omega} \frac{\kappa}{\omega^{2}+\kappa^{2}}
$$

In the limit $\mu \rightarrow \infty$ we expand the second factor in Eq. (29) in powers of $\omega^{2} / \mu^{2}$ and find $R_{A}=1-\left\langle\omega^{2}\right\rangle / \mu^{2}$.

For the Gaussian spectrum the threshold is at $\mu_{0}=$ $\sqrt{2 / \pi} \approx 0.7979$. The spectrum has unit variance, so at large $\mu$ we have $R_{A}=1-\mu^{-2}$. Integrating Eq. (29) using Eqs. (22) and (23) with $\theta=\pi / 4$ and $\Omega=\mathrm{i} \kappa$ we find

$$
\frac{1}{\mu}=\sqrt{\frac{\pi}{2}} \exp \left(\frac{\kappa^{2}}{2}\right) \operatorname{erfc}\left(\frac{\kappa}{\sqrt{2}}\right) .
$$

We thus have a parametric solution for $R_{A}(\mu)$ in the form $\mu(\kappa)$ and $R_{A}(\kappa)=\kappa / \mu(\kappa)$, shown in Fig. 6. For the box spectrum, the threshold is at $\mu_{0}=2 / \pi \approx 0.6366$ and

$$
R_{A}=\mu^{-1} \operatorname{cotan}\left(\mu^{-1}\right)
$$

We show this result as a red, dashed line in Fig. 6. 


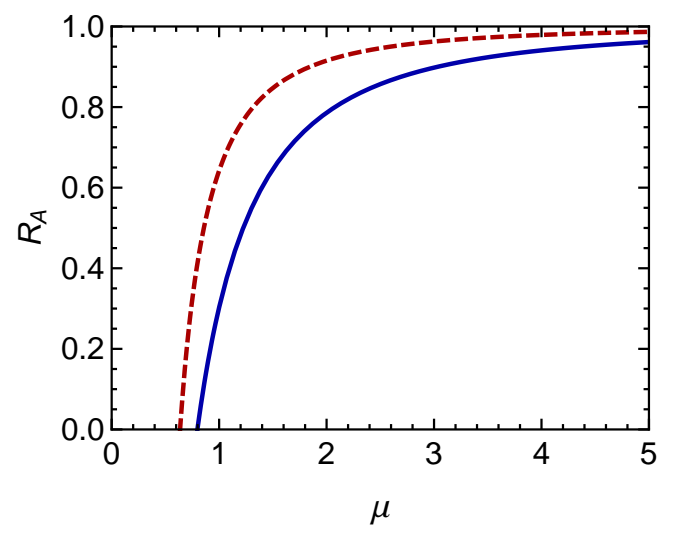

FIG. 6: Asymptotic order parameter $R_{A}$ for the Gaussian spectrum (blue, solid) and the box spectrum (red, dashed).

\section{B. Discrete polarization vectors}

When the system develops a collective mode, it settles only slowly to the final state of a pure precession, maintaining a long-lasting collective oscillation around the asymptotic solution. This observation suggests that the phase transition to a non-vanishing average $\langle P\rangle$ may already occur in a simpler system. To investigate this question we represent the spectrum schematically by two discrete polarization vectors, one for the negative and one for the positive frequency modes, respectively. To be specific, we use the normalized symmetric spectrum

$$
g_{\omega}=\frac{\delta\left(\omega+\frac{1}{2}\right)+\delta\left(\omega-\frac{1}{2}\right)}{2},
$$

and maximal mixing. This coarse representation of the box spectrum does not allow for decoherence, yet we may study its evolution and ask for its eigenmodes as a function of the interaction strength.

The symmetries of our problem imply that $\mathbf{P}(t)$ has only one nonvanishing component $P(t)$. From the full EoMs we find an equation of the form "kinetic plus potential energy is constant" $\frac{1}{2} \dot{P}^{2}+V(P)=0$ with

$$
V(P)=\frac{\mu^{2}\left(1-P^{2}\right)^{2}-\left(1-P^{2}\right)}{2} .
$$

We show this potential in Fig. 7 for several values of $\mu$.

The motion starts at $P=1$ with total energy 0 . For $0 \leq \mu<1$ the motion oscillates between $-1 \leq P \leq+1$ and $\langle P\rangle=0$. On the other hand, for $\mu>1$ the system is trapped in the region of positive $P$. It oscillates in the range $P_{\min }<P<1$ around the average $\langle P\rangle=\frac{1}{2}\left(1+P_{\min }\right)$. The turning point is $P_{\min }=$ $\sqrt{1-\mu^{-2}}$ so that

$$
\langle P\rangle=\frac{1+\sqrt{1-\mu^{-2}}}{2} .
$$

For $\mu<0$ we have $\langle P\rangle=0$ and thus we find the overall behavior shown in Fig. 8 ,

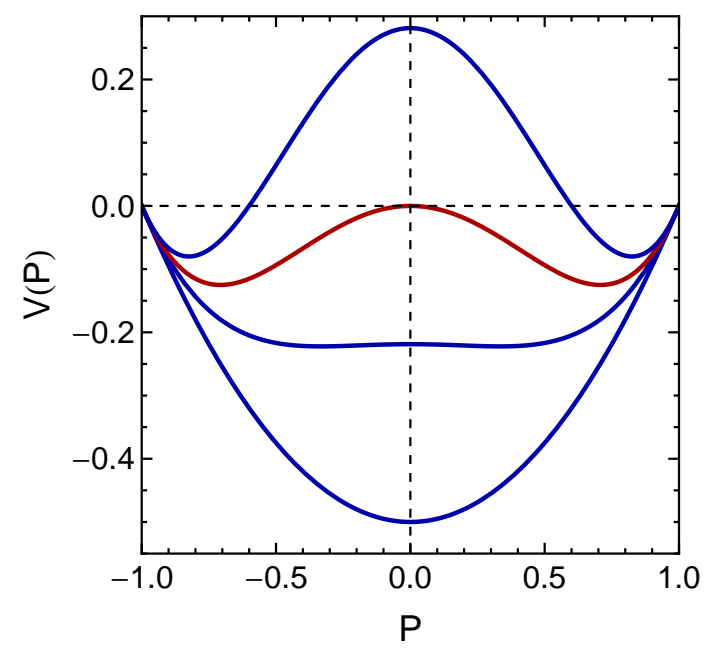

FIG. 7: Potential $V(P)$ defined in Eq. (33) for $\mu=0, \frac{3}{4}, 1$, and $\frac{5}{4}$ (bottom to top).

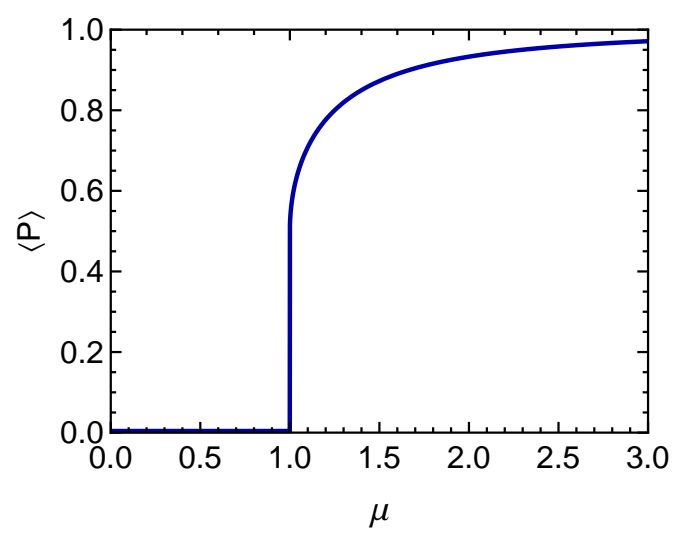

FIG. 8: $\langle P\rangle$ given by Eq. (34) for the case of two discrete polarization vectors.

The simple system of two discrete polarization vectors nicely reproduces the key features observed for a continuous spectrum. In particular, the threshold behavior is primarily related to the global properties of the selfinteracting system. Decoherence toward the asymptotic $P(t)$ is a secondary effect.

\section{RESONANT DECOHERENCE}

\section{A. Vanishing mixing angle}

For non-maximal mixing, new phenomena can arise. We continue to use a symmetric spectrum $g_{\omega}$, but the co-rotation frequency for $\mu<\infty$ is no longer identical with the synchronization frequency $\omega_{\text {sync }}=0$. As an extreme case we consider the limit of vanishing mixing angle. One may begin with the self-consistency conditions in the form of Eq. (18). In the limit $\theta \rightarrow 0$, up to linear order in $\theta$, we have $t^{2}=\tan ^{2}(2 \theta) \rightarrow 0$ and 


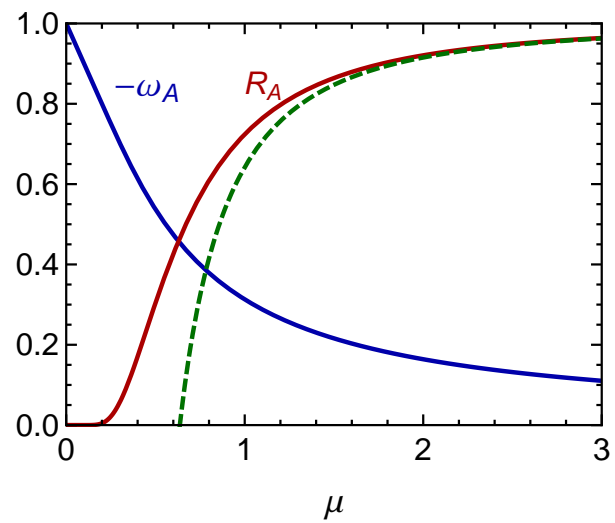

FIG. 9: Asymptotic values for global precession frequency $\omega_{A}$ (blue) and order parameter $R_{A}$ (red), assuming our box spectrum with vanishing mixing angle. The green (dashed) curve is $R_{A}$ for maximal mixing.

$$
\begin{aligned}
& c=\cos 2 \theta \rightarrow 1 \text { and the conditions become } \\
& 1=\int \mathrm{d} \omega g_{\omega} \frac{\mu^{2} R_{A}}{\left(\omega-\omega_{\mathrm{r}}\right)^{2}} \text { and } 1=\int \mathrm{d} \omega g_{\omega} \frac{\mu}{\omega-\omega_{\mathrm{r}}} .
\end{aligned}
$$

The second integral does not depend on $R_{A}$ and thus can be solved and inverted to obtain $\omega_{\mathrm{r}}(\mu)$. The result can be inserted in the first integral to find $R_{A}(\mu)$. For the box spectrum we find explicitly

$$
\omega_{\mathrm{r}}(\mu)=-\operatorname{coth}\left(\mu^{-1}\right) \quad \text { and } \quad R_{A}(\mu)=\frac{\operatorname{coth}^{2}\left(\mu^{-1}\right)-1}{\mu^{2}} .
$$

These results also follow from Eq. (23) in the $s \rightarrow 0$ limit. We show $-\omega_{\mathrm{A}}(\mu)$ and $R_{A}(\mu)$ in Fig. 9. Note that $-1<\omega_{\mathrm{A}}<0$ and in particular $\omega_{A} \rightarrow \omega_{\text {sync }}=0$ for $\mu \rightarrow \infty$. For small $\mu$ the function $R_{A}(\mu)$ is very small, but there is no threshold.

\section{B. Self-induced decoherence}

Inspecting Eq. (35) we notice that the first integral diverges if $g_{\omega}>0$ for $\omega=\omega_{\mathrm{r}}$, whereas the second remains meaningful in the principal-value sense. In other words, $\omega_{\mathrm{r}}$ can be determined from the second equation, whereas the first implies $R_{A}=0$, i.e. complete decoherence for any $\mu$. This conclusion applies to any spectrum with infinite tails, whereas the resonance frequency is outside of the box spectrum for any $\mu$.

To understand better the impact of the resonance, we return to the symmetric normalized box spectrum, augmenting it with small "wings"

$$
g_{\omega}= \begin{cases}(1-b) / 2 & \text { for }|\omega|<1 \\ b / 2 & \text { for } 1<|\omega|<2 \\ 0 & \text { otherwise }\end{cases}
$$

where $0<b \ll 1$. The self-consistency conditions imply qualitatively different solutions for any nonzero $b$ if $\omega_{\mathrm{r}}$,

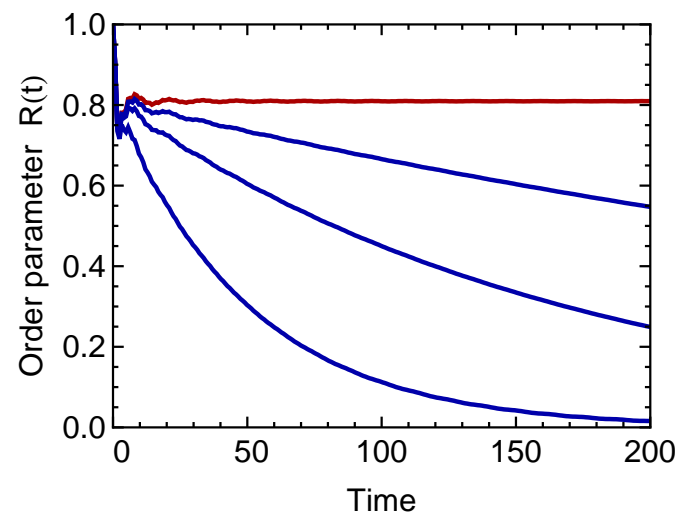

FIG. 10: Evolution of the order parameter for the box spectrum with wings of Eq. (37), using $b=0,10^{-3}, 3 \times 10^{-3}$, and $10^{-2}$ (top to bottom).

which is essentially fixed by the main part of the box, falls within the wings. On the other hand, if $b \ll 1$ the evolution of $P(t)$ can not at first differ from the case $b=0$, so the impact of a small $b$ can only manifest itself at late times.

We study numerically an example where the resonance frequency is fixed to fall into the center of the negativefrequency wing, i.e. $\omega_{\mathrm{r}}=-1.5$, implying with Eq. (36) $\mu=1 / \operatorname{acoth}(3 / 2)=2 / \log (5) \approx 1.243$ for $b \ll 1$. The evolution of $R(t)$ is shown in Fig. 10. For nonvanishing $b$ it is exponentially damped, the rate depending on $b$.

We also consider the late-time orientation of $\mathbf{P}_{\omega}$ relative to $\mathbf{B}$. When $\mathbf{P}_{\perp}$ has shrunk to zero, the remaining motion of $\mathbf{P}_{\omega}$ is a precession around the $z$-direction with a fixed angle. In Fig. 11] we show $\alpha_{\omega}=P_{\omega, \perp} /\left(g_{\omega} \sin 2 \theta\right)$, a quantity that is initially unity when all polarization vectors begin in the weak-interaction direction. We see that indeed the polarization vectors near $\omega_{\mathrm{r}}=-1.5$ have moved far away, whereas those with larger frequencies have actually been pulled closer to $\mathbf{B}$. We show the re-

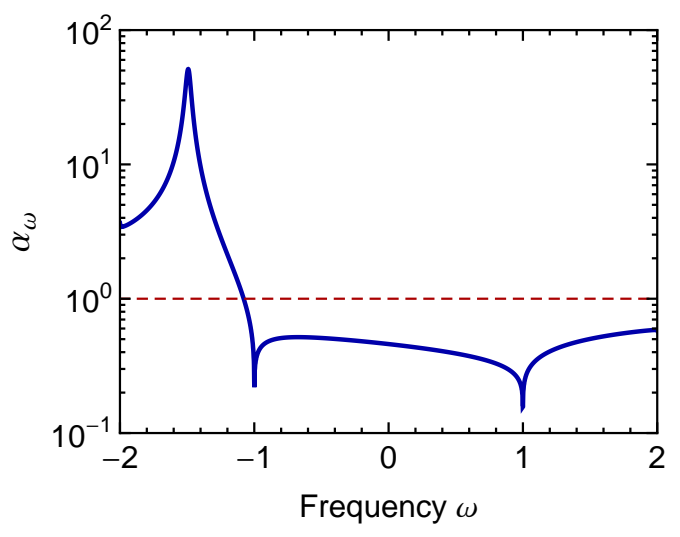

FIG. 11: Final deviation of polarization vectors from the $\mathbf{B}$ direction in units of $\sin 2 \theta$. The box spectrum with wings was used with $b=10^{-2}$. In the sudden approximation one would expect $\alpha_{\omega}=1$ (dashed line). 
sult for $b=10^{-2}$. For other values the curve looks the same, except that the resonant peak is taller for smaller $b$. For other spectra (e.g. Gaussian), the curve is different in detail, but there is always the resonance peak.

\section{Intermediate mixing angle}

When the mixing angle is not vanishingly small, the polarization vectors cannot be driven arbitrarily far away from the $\mathbf{B}$ direction. For example, $\mathbf{P}_{\omega}$ exactly on resonance will completely reverse and then come back and so forth. For example, the resonance peak in Fig. 11 reaches roughly up to $P_{\omega, \perp}=50 g_{\omega} \sin 2 \theta$. Therefore, if $\sin 2 \theta>0.02$, it is no longer geometrically possible to get that far and the resonance can not fully develop.

The threshold condition for partial synchronization of Eq. (26) here reads $s_{0}=\pi \mu b / 2$, assuming $\mu$ is such that the resonance falls into the wing. Specifically we consider $\mu=2 / \log (5) \approx 1.243$ and $b=10^{-2}$. With these numbers we expect complete decoherence for $\sin 2 \theta<0.01952$, in perfect agreement with our simple geometric estimate.

The different forms of evolution are illustrated in Fig. 12 showing the early evolution for the box with wings $(b=0.01$ and $\sin 2 \theta=0.04)$ in comparison with the simple box. The evolution is a two-step process, quickly decohering kinematically to what would be the final level for the simple box, and then decaying exponentially to the final level relevant when the spectrum has a wing.

For a spectrum with a long tail such as the Gaussian spectrum, the resonance always falls into the spectral range. In the $\sin 2 \theta=0$ limit one can not obtain stable synchronized oscillations - eventually the system decoheres, no matter how large $\mu$, although the exponential decay is very slow for large $\mu$. For nonvanishing mixing there exists a threshold so that for $\mu>\mu_{0}$ partial synchronization arises. In Fig. 13 we show the Gaussian $R_{A}(\mu)$ for several mixing angles. In contrast to the box spectrum, there is always a threshold and it moves to larger $\mu$ as expected.

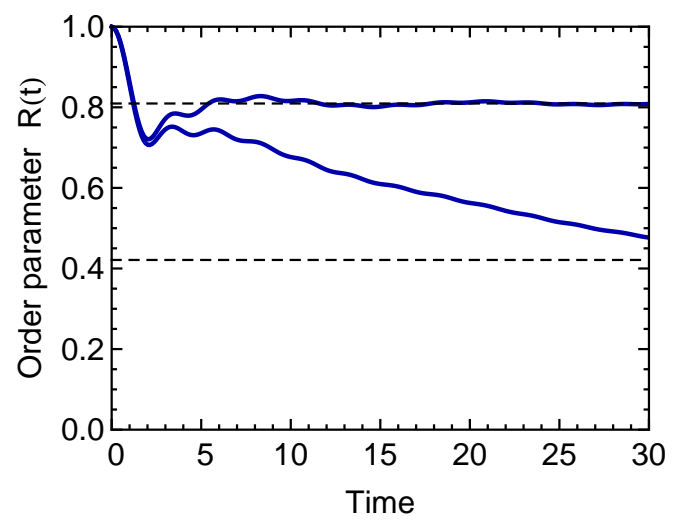

FIG. 12: Evolution of order parameter for box spectrum with $\sin 2 \theta=0.04$. Top curve: Simple box $(b=0)$. Bottom curve: $b=0.01$. Dashed lines show the asymptotic values.

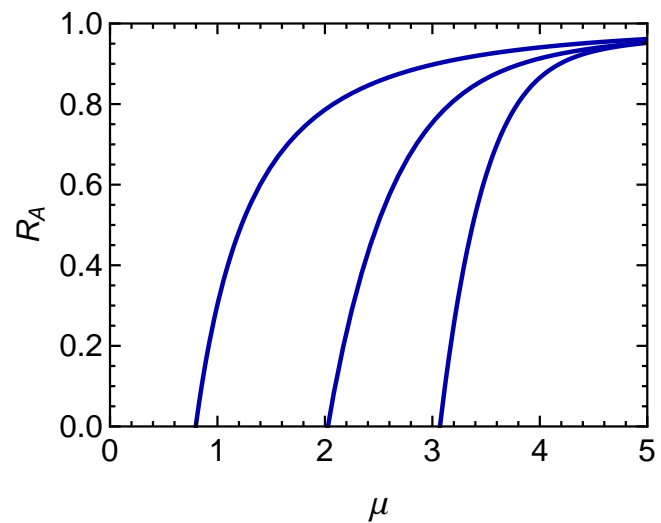

FIG. 13: Asymptotic order parameter for Gauss spectrum with mixing angles $\sin 2 \theta=1,0.1$ and 0.01 (left to right).

\section{CONCLUSIONS}

We have investigated the most elementary case of collective neutrino oscillations, an isotropic gas prepared in a single flavor with a fixed density. The seemingly simple question of partial synchronization for a large but finite density reveals a rich variety of possible solutions.

The idea of a late-time asymptotic solution has led us to formulate self-consistency relations that can be evaluated in the sudden approximation and that provide the asymptotic solution from the spectrum alone. We find excellent agreement of the asymptotic solutions such predicted with those from a numerical solution of the full EoMs. The agreement persists even when the true solution does not resemble the sudden approximation and we suspect that these relations are exact.

Partial synchronization requires a minimum density (a minimum effective interaction strength $\mu$ ). For smaller densities, decoherence is complete. For larger $\mu$, synchronization is partial and becomes perfect for $\mu \rightarrow \infty$. This "phase transition behavior" derives from a simple model where one coarse-grains the spectrum in terms of two polarization vectors, representing two halves of the spectrum. The system is represented by a particle moving in a double-well potential, and depending on $\mu$ it is trapped in one well or traverses both.

Non-maximal mixing enables a parametric resonance where those polarization vectors near the resonance frequency are driven away from their initial orientation. The back-reaction on the system consists of decoherence. This effect can only happen if the spectrum actually includes the resonance frequency, but this is always the case for spectra with long tails. The exact outcome depends on the spectrum and the mixing angle.

Decoherence can thus arise in different ways. In the dilute limit it consists of purely kinematical de-phasing of different modes. In the interacting system the effect is similar, except that each mode precesses around a different direction and this direction changes as the system decoheres. The self-induced resonant decoherence effect 
that we have discovered, on the other hand, is dynamical in the sense that an internal resonance of the system leads to an exponential decay of the global polarization vector. This decay can be slow, depending on parameters, and introduces a new time scale.

Our study is complementary to Pantaleone [2] who used the completely incoherent state as an initial condition. Based on an analytic argument, Pantaleone found that such a system will never develop spontaneous polarization ("neutral stability"). He also argued that a collective mode can be supported for any strength of $\mu$, in seeming contrast to our finding of a minimum required $\mu$ to maintain partial coherence. However, this question depends on the assumed initial condition. Beginning with all polarization vectors aligned with each other (our case), indeed a minimum $\mu$ is required for a coherent motion to survive. On the other hand, one can set up a pure precession mode for any strength of $\mu$ where all po- larization vectors are arranged in a co-rotating plane, not along a common direction [15, 16]. In practice this can be achieved by beginning with $\mu \rightarrow \infty$ (synchronized limit) and then reduce $\mu$ adiabatically to any desired value. This procedure leads to a spectral split if $\mu$ is reduced all the way to zero, and to a pure precession mode for any non-vanishing $\mu$.

\section{Acknowledgements}

This work was partly supported by the Deutsche Forschungsgemeinschaft under grant TR-27 "Neutrinos and Beyond," the Cluster of Excellence "Origin and Structure of the Universe" (Munich and Garching), and by the Italian MIUR and INFN through the "Astroparticle Physics" research project.
[1] J. Pantaleone, "Neutrino oscillations at high densities," Phys. Lett. B 287, 128 (1992).

[2] J. Pantaleone, "Stability of incoherence in an isotropic gas of oscillating neutrinos," Phys. Rev. D 58, 073002 (1998).

[3] S. Samuel, "Neutrino oscillations in dense neutrino gases," Phys. Rev. D 48, 1462 (1993).

[4] V. A. Kostelecký and S. Samuel, "Neutrino oscillations in the early universe with an inverted neutrino mass hierarchy," Phys. Lett. B 318, 127 (1993).

[5] V. A. Kostelecký and S. Samuel, "Self-maintained coherent oscillations in dense neutrino gases," Phys. Rev. D 52, 621 (1995).

[6] S. Samuel, "Bimodal coherence in dense selfinteracting neutrino gases," Phys. Rev. D 53, 5382 (1996).

[7] S. Pastor, G. G. Raffelt and D. V. Semikoz, "Physics of synchronized neutrino oscillations caused by self-interactions," Phys. Rev. D 65, 053011 (2002).

[8] Y. Y. Y. Wong, "Analytical treatment of neutrino asymmetry equilibration from flavour oscillations in the early universe," Phys. Rev. D 66, 025015 (2002).

[9] H. Duan, G. M. Fuller and Y.-Z. Qian, "Collective neutrino flavor transformation in supernovae," Phys. Rev. D 74, 123004 (2006).

[10] H. Duan, G. M. Fuller, J. Carlson and Y. Z. Qian, "Simulation of coherent non-linear neutrino flavor transformation in the supernova environment. I: Correlated neutrino trajectories," Phys. Rev. D 74, 105014 (2006).

[11] G. G. Raffelt and G. Sigl, "Self-induced decoherence in dense neutrino gases," Phys. Rev. D 75, 083002 (2007).

[12] R. F. Sawyer, "The multi-angle instability in dense neutrino systems," Phys. Rev. D 79, 105003 (2009).

[13] A. Esteban-Pretel, S. Pastor, R. Tomàs, G. G. Raffelt and G. Sigl, "Decoherence in supernova neutrino transformations suppressed by deleptonization," Phys. Rev. D 76, 125018 (2007).

[14] S. Hannestad, G. G. Raffelt, G. Sigl and Y. Y. Y. Wong, "Self-induced conversion in dense neutrino gases: Pendulum in flavor space," Phys. Rev. D 74, 105010 (2006); Erratum ibid. 76, 029901 (2007).

[15] G. G. Raffelt and A. Yu. Smirnov, "Self-induced spectral splits in supernova neutrino fluxes," Phys. Rev. D 76, 081301 (2007); Erratum ibid. 77, 029903 (2008).

[16] G. G. Raffelt and A. Yu. Smirnov, "Adiabaticity and spectral splits in collective neutrino transformations," Phys. Rev. D 76, 125008 (2007).

[17] B. Dasgupta, A. Dighe, G. G. Raffelt and A. Yu. Smirnov, "Multiple spectral splits of supernova neutrinos," Phys. Rev. Lett. 103, 051105 (2009).

[18] H. Duan, G. M. Fuller and Y. Z. Qian, "Collective neutrino oscillations," Annu. Rev. Nucl. Part. Sci. 60, 569 (2010).

[19] A. D. Dolgov, "Neutrinos in the early universe," Sov. J. Nucl. Phys. 33, 700 (1981) [Yad. Fiz. 33, 1309 (1981)].

[20] G. Sigl and G. Raffelt, "General kinetic description of relativistic mixed neutrinos," Nucl. Phys. B 406, 423 (1993). 